\title{
Optical absorption in paired correlated random lattices
}

\author{
Francisco Domínguez-Adame and Enrique Maciá* \\ Departamento de Física de Materiales, Facultad de Físicas, Universidad Complutense, E-28040 Madrid, Spain
}

\begin{abstract}
Angel Sánchez
Theoretical Division and Center for Nonlinear Studies, Los Alamos National Laboratory, Los Alamos, New Mexico 87545 and Escuela Politécnica Superior, Universidad Carlos III de Madrid, C./ Butarque, 15, E-28911 Leganés, Madrid, Spain
\end{abstract}

(Received 11 February 1994)

\begin{abstract}
Optical absorption in a random one-dimensional lattice in the presence of paired correlated disorder is studied. The absorption line shape is evaluated by solving the microscopic equations of motion of the Frenkel-exciton problem in the lattice. We find that paired correlation causes the occurrence of well-defined characteristic lines in the absorption spectra which clearly differ from the case of unpaired correlation. The behavior of the absorption lines as a function of defect concentration is studied in detail. We also show how exciton dynamics can be inferred from experimental data by deriving an analytical expression relating the energy and intensity of lines to the model parameters.
\end{abstract}

\section{INTRODUCTION}

During the past few years, it is being realized that correlated disorder has profound effects in quasiparticle dynamics and produces a variety of complex and new phenomena. The notion of correlated disorder implies that certain physical parameters in random systems are not completely independent within a given correlation length, thus leading to a competition between shortrange order effects and the underlying long-range disorder. It is well known, for instance, that delocalized electrons $^{1-4}$ and vibrations ${ }^{5}$ appear in one-dimensional random systems with correlated disorder. Following the above line of research, we have recently carried out the time-domain analysis of trapping of Frenkel excitons in one-dimensional systems with paired correlated traps, randomly placed in an otherwise perfect lattice. ${ }^{6}$ Results were compared with the dynamics of one-dimensional lattices whose excited states are also Frenkel excitons, but in which transport is affected by the presence of unpaired traps. ${ }^{7}$ We found that pairing of traps leads to a slowdown of the trapping rate due to the occurrence of larger segments of the lattice which are free of traps. In view of these results, the question arises as to whether the fact that disorder is correlated will affect the opticalabsorption spectrum of the lattice. If so, it would be interesting to find a relationship between the characteristic absorption lines and the model parameters in order to compare with experimental data. In this paper we aim to answer these questions by numerical simulation of optical spectra. We believe that the interest of this work is beyond the formal study of the exciton dynamics: Indeed, our treatment may help describe the optical properties of some polymers with active molecules, provided that interchain interactions become much smaller that intrachain interactions so that a one-dimensional model can be applied as a first approximation.

\section{MODEL}

The Hamiltonian for the Frenkel-exciton problem can be written in the tight-binding form with nearestneighbor interactions as follows (we use units such that $\hbar=1)$ :

$$
\mathcal{H}=\sum_{k} V_{k} a_{k}^{\dagger} a_{k}+T \sum_{k}\left(a_{k}^{\dagger} a_{k+1}+a_{k+1}^{\dagger} a_{k}\right) .
$$

Here $a_{k}$ and $a_{k}^{\dagger}$ are the exciton annihilation and creation operators, respectively. $V_{k}$ is the transition frequency at site $k$ and $T$ is the nearest-neighbor coupling, which is assumed to be constant in the whole lattice. The paired correlated disorder is introduced as follows: Transition frequencies can only take on two values, $V$ and $V^{\prime}$, with the additional constraint that $V^{\prime}$ appears only in pairs of neighboring sites (the correlation length is roughly the lattice spacing). Such a pair of sites will be called a dimer defect. In addition, we define the defect concentration $c$ as the ratio between the number of sites with transition frequency $V^{\prime}$ and the total number of sites in the lattice.

The line shape $I(E)$ of an optical-absorption process in which a single exciton is created in a lattice with $N$ sites is given by (see Ref. 8 for details)

$$
I(E)=-\frac{2}{\pi N} \int_{0}^{\infty} d t e^{-\alpha t} \sin (E t) \operatorname{Im}\left(\sum_{k} G_{k}(t)\right),
$$

where the factor $\exp (-\alpha t)$ takes into account the broadening due to the Lorentzian instrumental resolution function of width $\alpha$. The correlation functions $G_{k}(t)$ obey the equations of motion

$$
i \frac{d}{d t} G_{k}(t)=\sum_{j} H_{k j} G_{j}(t)
$$


with the initial conditions $G_{k}(0)=1$. The diagonal elements of the tridiagonal matrix $H_{k j}$ are $V_{k}$ whereas offdiagonal elements are simply given by $T$. Once these equations of motion are solved, the line shape is found using Eq. (2).

\section{RESULTS AND DISCUSSIONS}

We have numerically solved the equation of motion (3) for chains of $N=2000$ sites using an implicit integration scheme. In order to minimize end effects, spatial periodic boundary conditions are introduced. Once the functions $G_{k}(t)$ are known, the line shape $I(E)$ is evaluated by means of (2). Energy will be measured in units of $T$ whereas time will be expressed in units of $T^{-1}$. Since we are mainly interested in the effects due to the presence of correlated disorder in the random system rather than in the effects of the different parameters on the optical-absorption process, we will fix the values of $V, V^{\prime}$, and $T$, focusing our attention on the defect concentration $c$. Thus, we have set $V=4, V^{\prime}=10$, and $T=-1$ henceafter as representative values. The width of the instrumental resolution was $\alpha=0.5$. The maximum integration time and the integration time step were 16 and $8 \times 10^{-3}$, respectively; larger maximum integration times or smaller time steps led to the same general results. The defect concentration $c$ ranged from 0.01 up to 0.9 and for each lattice realization a random distribution of dimer defects was chosen. In addition, lattices with unpaired defects have been studied and compared with lattices containing the same fraction of paired defects. This enables us to separate the effects merely due to optical absorption in one dimension from those which manifest the peculiarities of the correlation between random defects.

In the absence of defects the spectrum is a single Lorentzian line centered at $E=V+2 T$, which with our choice of parameters is $E=2$. When defects are introduced in the lattice, a broadening of this main line is observed accompanied by a shift of its position to higher energies on increasing defect concentration. Keeping these general results in mind we now proceed to discuss the main features of the spectra obtained when defects are present in the system. The obtained results are shown in Figs. 1-3, which respectively correspond to an increasing defect concentration. From a close inspection of these figures several conclusions can be drawn. First of all, we observe that, in addition to the main absorption line located close to $E=2$, several satellites appear in the spectra. These satellites become more intense as the defect concentration increases. Such lines are related to the presence of defects in the lattice, and their positions only depend on the particular kind of disorder (paired or unpaired). On increasing defect concentration, the highenergy lines increase at the expenses of the main line (compare Figs. 1-3). For low defect concentration (see Fig. 1), two lines appear at about $E=9.0$ and $E=10.2$, whose positions are almost independent of $c$. The line at $E=9.0$ is more intense for lattices with dimer defects whereas the line at $E=10.2$ is weaker in that case. In ad-

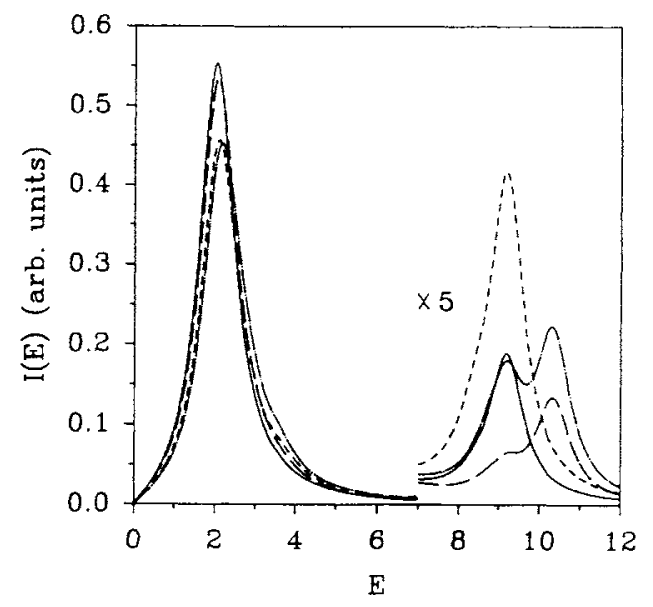

FIG. 1. Absorption spectra for one-dimensional random lattices with concentration of paired defects $c=0.1$ (solid line) and $c=0.2$ (short-dashed line). Results are compared to lattices with the same concentration of unpaired defects $c=0.1$ (long-dashed line) and $c=0.2$ (dot-dashed line). All spectra have the same area. Note the magnification factor of the high-energy part of the spectra.

dition, a small shoulder appears at about $E=3.8$, and it becomes more intense in the case of unpaired lattices. On increasing defect concentration, this shoulder is clearly resolved and a new line develops at around $E=8.5$ (see Fig. 2). The intensity of this new line rapidly increases with $c$ and shifts to the low-energy region of the spectra (see Fig. 3), until it is placed close to $E=V^{\prime}+2 T$ ( $E=8$ with the chosen parameters).

First we discuss the shift of the main absorption line toward higher energies on increasing defect concentration. In the case of unpaired defects this shift $\Delta E$ depends linearly on defect concentration $c$; using a least squares fit we have found that $\Delta E \approx 0.92 c$ in the range $c=0.01$ up to $c=0.4$. This behavior is in agreement with the

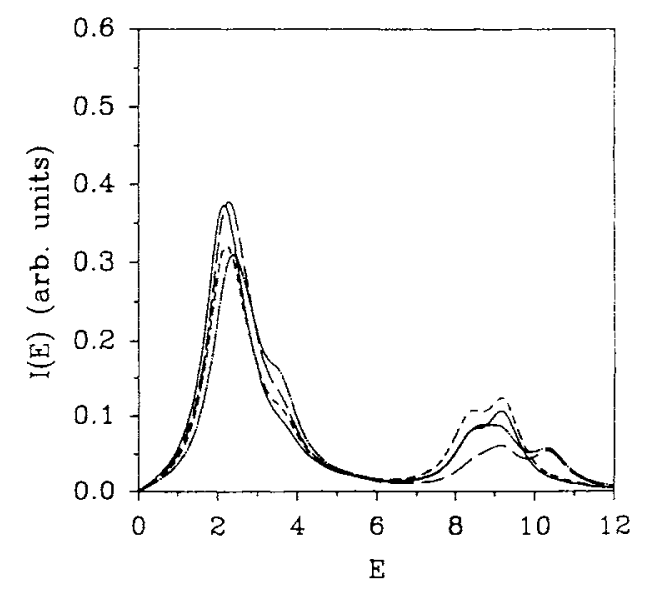

FIG. 2. Absorption spectra for one-dimensional random lattices with concentration of paired defects $c=0.3$ (solid line) and $c=0.4$ (short-dashed line). Results are compared to lattices with the same concentration of unpaired defects $c=0.3$ (long-dashed line) and $c=0.4$ (dot-dashed line). 


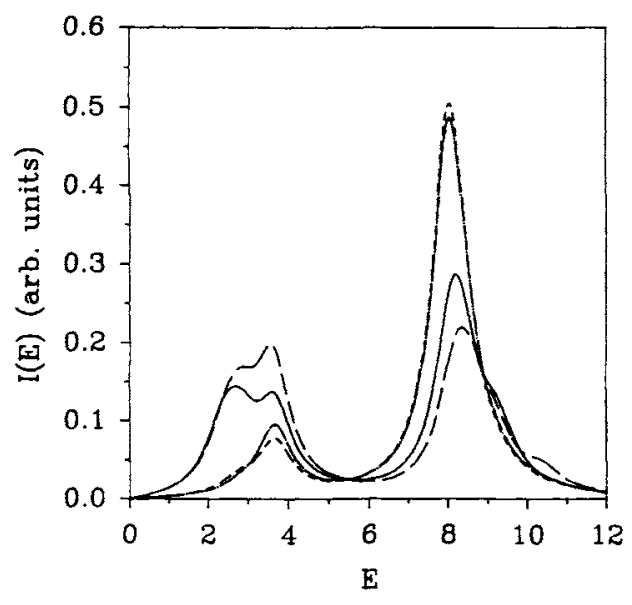

FIG. 3. Absorption spectra for one-dimensional random lattices with concentration of paired defects $c=0.7$ (solid line) and $c=0.9$ (short-dashed line). Results are compared to lattices with the same concentration of unpaired defects $c=0.7$ (long-dashed line) and $c=0.9$ (dot-dashed line).

average- $T$-matrix approximation (ATA), which predicts that the shift increases linearly with defect concentration in the absence of correlations. ${ }^{8}$ On the contrary, pairing of defects causes a quadratic dependence on the defect concentration of the form $\Delta E \approx 1.20 c^{2}$. It is worth mentioning that the line shift $\Delta E$ for the paired correlated lattice is always smaller than $\Delta E$ for the unpaired one, for the same defect concentration $c$ in the range considered. This is an important result since it suggests that pairing of defects reduces the effects of disorder on the Frenkel-exciton dynamics. The physical explanation of this behavior is based on the particular spatial distribution displayed by the defects when pairing is present. It becomes clear that the average length of segments which are free of defects is larger when correlation is present since defects always appear in pairs. Therefore, pairing of defects implies a smaller disruption of the lattice and consequently the random distribution of such defects causes less influence on the exciton dynamics.

Now we focus our attention on the satellite lines present in the spectra. In order to study the results from a quantitative point of view, we consider the following two center problem, which describes the optical absorption spectrum of two isolated, coupled defect sites:

$$
\begin{aligned}
& i \frac{d}{d t} G_{1}(t)=V_{1} G_{1}(t)+T G_{2}(t), \\
& i \frac{d}{d t} G_{2}(t)=V_{2} G_{2}(t)+T G_{1}(t) .
\end{aligned}
$$

These equations of motion can be easily solved using the initial conditions $G_{1}(0)=G_{2}(0)=1$. Inserting the result in (2) and taking $\alpha=0$ for the sake of simplicity, one obtains

$$
I(E)=I_{+} \delta\left(E-E_{+}\right)+I_{-} \delta\left(E-E_{-}\right)
$$

where the intensity of each component is given by

$$
I_{ \pm}=\frac{1}{2} \pm \frac{1}{2}\left[1+\left(\frac{V_{1}-V_{2}}{2 T}\right)^{2}\right]^{-1 / 2}
$$

and centered at

$$
E_{ \pm}=\frac{V_{1}+V_{2}}{2} \pm T \sqrt{1+\left(\frac{V_{1}-V_{2}}{2 T}\right)^{2}}
$$

From Eqs. (5) and (6) we obtain the following possible scenarios. If the two transition frequencies of defects are $V_{1}=V_{2}=V^{\prime}$, the intensity $I_{-}$vanishes due to the fact that only the dipole matrix element involving symmetric states contributes to the optical-absorption spectrum. In this case the spectrum exhibits a single line centered at $E_{+}=V^{\prime}+T=9.0$. On the contrary, if the transition frequencies are different (say, $V_{1}=V$ and $V_{2}=V^{\prime}$ ), the optical spectrum presents two components centered at $E_{ \pm}=\left(V+V^{\prime}\right) / 2 \pm T \sqrt{1+\left(V-V^{\prime}\right)^{2} / 4 T^{2}}=7 \mp \sqrt{10}$ so that $E_{-} \sim 10.2$ and $E_{+} \sim 3.8$.

With this analytical results at hand, the quantitative interpretation of the obtained spectra is straightforward since each absorption line can be related to a specific kind of defect in the lattice. We shall consider, in first place, the case of low defect concentration (Fig. 1). Besides the main line close to $E=2$, characteristic satellites are clearly visible at about $E=10.2$ (more intense in unpaired lattices) or $E=9.0$ (more intense in paired lattices). These values agree very well with our theoretical predictions shown above: The line at $E=10.2$ is related to centers $V_{1}=V$ and $V_{2}=V^{\prime}$, and the number of such centers is greater in unpaired lattices, whereas the line at $E=9.0$ is related to dimer defects and the probability of finding such defects in unpaired lattices is actually very small at low $c$. Analogous comments hold for the line centered at $E=3.8$ since it originates from the same defects as the line centered at $E=10.2$. On further increasing defect concentration to intermediate ranges $(c \sim 0.5)$ these characteristic lines continue to dominate the high-energy region of the absorption spectra (Fig. 2). Ultimately, in the limit $c \rightarrow 1$, the absorption spectrum is dominated again by a single line centered at $E=V^{\prime}+2 T=8$, as it corresponds for a lattice entirely composed of defect sites. This trend is clearly observed in our spectra for both unpaired and paired lattices (Fig. 3).

To seek further confirmation of the above explanations, we have investigated the intensity dependence on the defect concentration for the lines centered at $E=9.0$ and $E=10.2$. Results are shown in Fig. 4. The intensity of the line at $E=9.0$ increases almost linearly at low defect concentration in the case of paired lattices, as shown in Fig. 4(a). This is to be expected since such defects are introduced on purpose in the lattice. On the contrary, the intensity of this line increases quadratically with defect concentration in unpaired lattices since the probability of finding dimer defects in such uncorrelated lattices also increases as $c^{2}$. Concerning the line centered at $E=10.2$, its intensity increases sublinearly with defect concentration in the case of unpaired lattices, as shown in Fig. 4(b). This is due to the fact that on increasing defect con- 


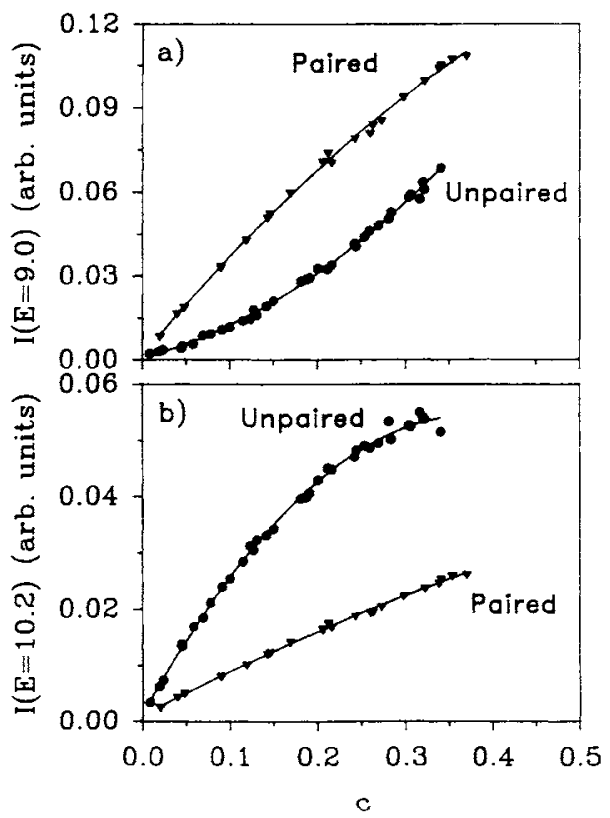

FIG. 4. Intensity of the absorption lines centered at (a) $E=9.0$ and (b) $E=10.2$ as a function of dimer defect concentration in random one-dimensional lattices with paired defects (solid triangles) and unpaired defects (solid circles). Solid lines indicate the best least squares fit.

centration dimer defects appear and, consequently, the number of centers $V_{1}=V$ and $V_{2}=V^{\prime}$ cannot increase linearly. In the case of paired lattices the intensity of this line increases almost linearly since the number of centers $V_{1}=V$ and $V_{2}=V^{\prime}$ equals the number of dimer defects whenever the probability of pairing of such defects remains small at low defect concentration. Therefore, we are led to conclude that our interpretation of all relevant lines in the optical spectra is correct, and those spectra show indeed clear differences when coming from paired or unpaired disorder.

\section{CONCLUSIONS}

In summary, we have studied the absorption spectrum corresponding to the Frenkel-exciton Hamiltonian for a random system in which a correlation length of the order of the lattice spacing is intentionally introduced, so that defects can only appear in pairs. By comparing the obtained spectra with those corresponding to the uncorrelated random system we are able to identify characteristic absorption lines which can be directly related to the presence of dimer defects. Furthermore, we have obtained analytical expressions which explain our synthetic spectra very well, and relate microscopic system parameters, like transition frequencies or defect concentration, to experimental data, like position of the lines. Our results show that the presence of correlation effects in random systems can be readily determined from analysis of the absorption spectra of these samples, following the lines of reasoning presented in this work.

\section{ACKNOWLEDGMENTS}

The authors thank Bianchi Méndez for a critical reading of the manuscript. This work is partially supported by UCM under project No. PR161/93-4811. A.S. is supported by a Ministerio de Educación y Ciencia (Spain)/Fulbright postdoctoral scholarship, by Dirección General de Investigación Científica y Técnica (Spain) through Project No. PB92-0248, and by the European Community (Union Human Capital and Mobility Programme through Contract No. ERBCHRXCT930413). Work at Los Alamos is performed under the auspices of the U.S. Department of Energy.
* Also at Instituto de Estudios Interdisciplinares, El Guijo, Z4 Galapagar, E-28260 Madrid, Spain.

${ }^{1}$ D. H. Dunlap, H.-L. Wu, and P. Phillips, Phys. Rev. Lett. 65, 88 (1990).

${ }^{2}$ H.-L. Wu and P. Phillips, Phys. Rev. Lett. 66, 1366 (1991).

${ }^{3}$ J. C. Flores and M. Hilke, J. Phys. A 26, L1255 (1993).

${ }^{4}$ A. Sánchez, E. Maciá, and F. Domínguez-Adame, Phys.
Rev. B 48, 147 (1994).

${ }^{5}$ F. Domínguez-Adame, E. Maciá, and A. Sánchez, Phys. Rev. B 48, 6054 (1993).

${ }^{6}$ F. Domínguez-Adame, B. Méndez, A. Sánchez, and E. Maciá, Phys. Rev. B 40, 3839 (1994).

${ }^{7}$ D. L. Huber and W. Y. Ching, Chem. Phys. 146, 409 (1990).

${ }^{8}$ D. L. Huber and W. Y. Ching, Phys. Rev. B 39, 8652 (1989). 